RESPIRATORY INFECTION

\title{
Severe acute respiratory syndrome: report of treatment and outcome after a major outbreak
}

\author{
J J Y Sung, A Wu, G M Joynt, K Y Yuen, N Lee, P K S Chan, C S Cockram, A T Ahuja, L M Yu, \\ V W Wong, D S C Hui
}

Thorax 2004;59:414-420. doi: 10.1136/thx.2003.014076

\begin{abstract}
See end of article for authors' affiliations

.....................

Correspondence to: Dr D S C Hui, Department of Medicine and

Therapeutics, Prince of Wales Hospital, Shatin NT, Hong Kong; dschui@ cuhk.edu.hk
\end{abstract}

Received 5 August 2003 Accepted

26 November 2003

\begin{abstract}
Background: The outcome is reported of a prospective uncontrolled study based on a stepwise treatment protocol during an outbreak of severe acute respiratory syndrome (SARS) in Hong Kong.

Method: One hundred and thirty eight patients were treated with broad spectrum antibiotics, a combination of ribavirin and low dose corticosteroid, and then intravenous high dose methylprednisolone according to responses. Sustained response to treatment was defined as (1) defervescence for $\geqslant 4$ consecutive days, (2) resolution of lung consolidation by $>25 \%$, and (3) oxygen independence by the fourth day without fever. Patients with defervescence who achieved either criterion 2 or 3 were classified as partial responders. Patients who fell short of criteria 2 and 3 were non-responders.

Results: Laboratory confirmation of SARS coronavirus infection was established in $132(95.7 \%)$. None responded to antibiotics but $25(18.1 \%)$ responded to ribavirin + low dose corticosteroid. Methylprednisolone was used in 107 patients, of whom $95(88.8 \%)$ responded favourably. Evidence of haemolytic anaemia was observed in 49 (36\%). A high level of C-reactive protein at presentation was the only independent predictor for use of methylprednisolone (odds ratio 2.18 per $10 \mathrm{mg} / \mathrm{dl}$ increase, $95 \%$ confidence interval 1.12 to $4.25, p=0.02)$. Thirty seven patients $(26.8 \%)$ required admission to the intensive care unit and $21(15.2 \%)$ required invasive mechanical ventilation. There were 15 deaths (mortality rate 10.9\%), most with significant co-morbidities, whereas 122 (88.4\%) had been discharged home 4 months after the outbreak onset.

Conclusion: The use of high dose pulse methylprednisolone during the clinical course of a SARS outbreak was associated with clinical improvement, but randomised controlled trials are needed to ascertain its efficacy in this condition.
\end{abstract}

1: March 2003 there was a serious outbreak of severe acute respiratory syndrome (SARS) in Hong Kong. One hundred and thirty eight patients and healthcare workers contracted the disease from a single index patient on a medical ward at the Prince of Wales Hospital. ${ }^{1}$ Patients in this cohort had a very similar presentation with fever, chills and rigor, myalgia, and progression to respiratory failure within l-2 weeks. Within a month the disease had spread to Singapore, Vietnam, Taiwan, Germany, Canada, and the United States. ${ }^{2}$

SARS is caused by a novel coronavirus $(\mathrm{CoV}),{ }^{3-5}$ probably originating from an animal reservoir. Without knowledge of the underlying cause of the disease during the outbreak, treatment of this newly emerging disease was empirical. A team of physicians from the Department of Medicine and Therapeutics (infectious disease, respiratory medicine and general medicine) and the intensive care unit was formed to manage the patients. A treatment protocol was developed in response to the demands of the event. The results are reported here.

\section{METHODS}

The data of our previously reported cohort ${ }^{1}$ recruited during the 2 week period from 11 March to 25 March 2003 were analysed. Because of the unexpected acute medical crisis with many healthcare workers becoming infected, a blind control study was not considered feasible. Instead, a stepwise management algorithm was developed through observation and discussion of the progress of the patients' clinical condition on a day-to-day basis. All data were prospectively collected to assist future clinical management decisions. The final clinical outcome is reported up to 28 July 2003.

\section{Diagnosis and monitoring of progress}

The CDC criteria for diagnosis of SARS were applied. ${ }^{6}$ Our case definitions were: (1) fever (temperature $>38^{\circ} \mathrm{C}$ ), (2) chest radiograph (plain radiograph and/or CT thorax) showing evidence of consolidation with or without respiratory symptoms such as cough or shortness of breath, and (3) history of exposure to an index case suspected of having SARS or direct contact with a person who fell ill following exposure to the index case.

Initial investigations included complete blood count (including differential count), clotting profile (PT, APTT, INR, D-dimer), C-reactive protein (CRP), and serum biochemistry (including electrolytes, urea and creatinine levels, serum alanine aminotransferase, creatine phosphokinase $(\mathrm{CPK})$, lactate dehydrogenase $(\mathrm{LDH}))$. These parameters, together with chest radiography and vital signs (blood pressure, pulse, respiratory rate), were monitored regularly.

Serological examination for SARS CoV infection was performed for all patients. The level of SARS CoV IgG antibody was measured by an immunofluorescence assay based on Vero cells infected with CoV isolated from a patient with SARS. Paired serum samples were tested at serial twofold dilutions starting from 1:40. The tests were regarded as indicating SARS CoV infection if a seroconversion or fourfold rise in antibody titre was detected. Viral isolation using Vero cell culture has been previously described. ${ }^{1}$

\section{Treatment protocol}

Patients were treated for the first 2 days with broad spectrum antibiotics for community acquired pneumonia according to the American Thoracic Society guidelines. ${ }^{7}$ Initial treatment 
consisted of intravenous cefotaxime $1 \mathrm{~g}$ every 6 hours with either oral levofloxacin $500 \mathrm{mg}$ daily or clarithromycin $500 \mathrm{mg}$ twice daily. Oseltamivir was also given to the initial patients to treat possible influenza infection (fig 1). Clinical symptoms, blood oxygen saturation, and the chest radiograph were assessed daily. If fever persisted after 48 hours, patients were given a combination of ribavirin and "low dose" corticosteroid therapy commencing on day 3-4 (oral ribavirin as a loading dose of $2.4 \mathrm{~g}$ stat followed by $1.2 \mathrm{~g}$ three times daily and prednisolone $0.5-1 \mathrm{mg} / \mathrm{kg}$ body weight per day), whereas those with dyspnoea were treated with intravenous ribavirin (400 mg every 8 hours) combined with hydrocortisone ( $100 \mathrm{mg}$ every 8 hours). Pulses of high dose methylprednisolone $(0.5 \mathrm{~g}$ intravenous infusion for three consecutive days) were given as a response to persistence or recurrence of fever and radiographic progression of lung opacity with or without hypoxaemia despite initial combination therapy. Further pulses of methylprednisolone were given if there was no clinical or radiological improvement, up to a total of $3 \mathrm{~g}$. The intention was to continue with the combination of ribavirin and "low dose" corticosteroid for up to 12 days when there was complete resolution of lung opacity. Those who became afebrile but with incomplete radiological resolution were given oral ribavirin $600 \mathrm{mg}$ three times daily and prednisolone $0.5 \mathrm{mg} / \mathrm{kg}$ body weight per day for at least one further week.

\section{Intensive care and mechanical ventilation}

Patients who developed hypoxaemia were given supplemental oxygen. Patients were admitted to the intensive care unit (ICU) when severe respiratory failure developed as evidenced by (1) failure to maintain an arterial oxygen saturation of at least $90 \%$ while receiving supplemental oxygen of $50 \%$ and/or (2) respiratory rate $>35$ breaths/min. Non-invasive positive pressure ventilation was avoided because of the risk of viral transmission from mask leakage and flow compensation,

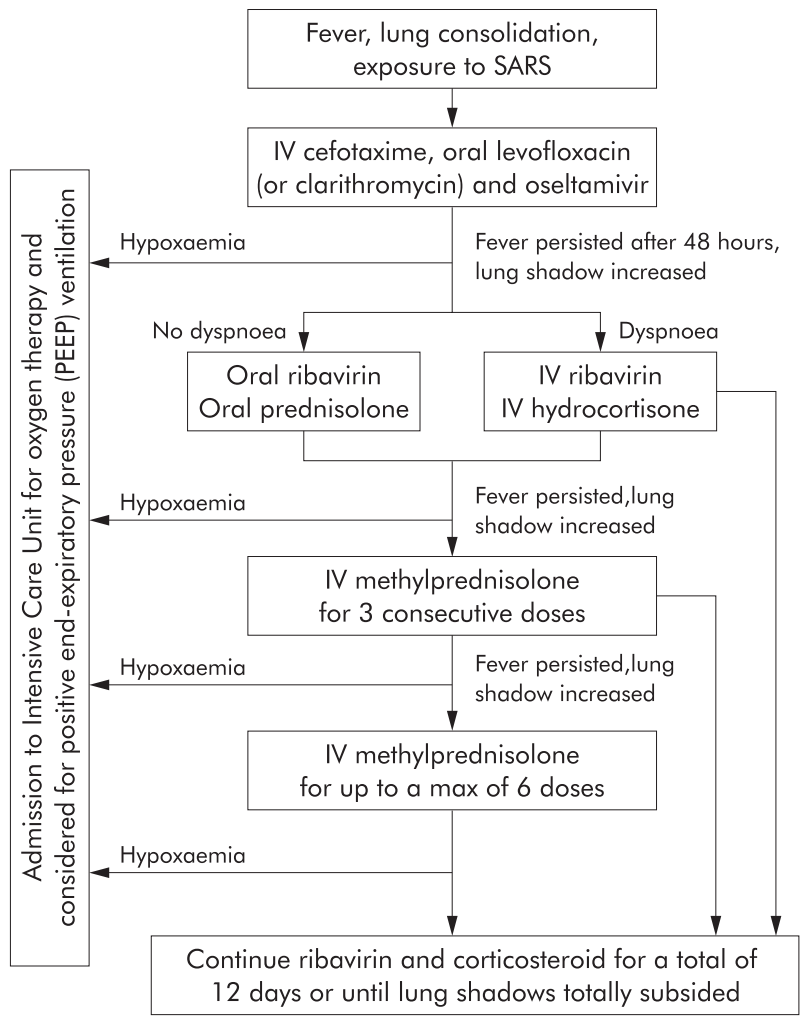

Figure 1 Treatment protocol for SARS. possibly causing wide dispersion of contaminated aerosol. Criteria for intubation and positive pressure ventilation were (1) persistent failure to achieve arterial oxygen saturation of $90 \%$ while receiving $100 \%$ oxygen via a non-rebreathing mask and/or (2) onset of respiratory muscle fatigue as evidenced by an increase in $\mathrm{PaCO}_{2}$, sweating, tachycardia, and/or a subjective feeling of exhaustion. Mechanical ventilation with synchronised intermittent mandatory ventilation or pressure control ventilation was instituted. Positive end expiratory pressure and inspired oxygen concentration were titrated to achieve an arterial saturation of $90-95 \%$. Tidal volume was maintained at $6-8 \mathrm{ml} / \mathrm{kg}$ estimated body weight and plateau pressure maintained at $30 \mathrm{~cm} \mathrm{H}_{2} \mathrm{O}$ or less. $\mathrm{PaCO}_{2}$ was allowed to rise provided the $\mathrm{pH}$ was $>7.15{ }^{8}$ Patients unable to meet the above parameters were ventilated in the prone position.

\section{Definitions of clinical outcome}

Sustained response (SR) to treatment was defined as (1) defervescence (daily peak temperature $\leqslant 37.5^{\circ} \mathrm{C}$ ) for at least four consecutive days; (2) radiological improvement as assessed by three radiologists who were blind to the clinical data of more than 25\%; and (3) oxygen independence as assessed by pulse oximetry $\left(\mathrm{SaO}_{2} \geqslant 95 \%\right.$ on room air) on the fourth afebrile day. Patients with defervescence who achieved either resolution of lung consolidation or oxygen independence, but not both, were classified as showing a partial response (PR). Patients who fell short of criteria 2 and 3 above were classified as non-responders to treatment (NR).

\section{Statistical analysis}

Demographic, clinical, laboratory and radiological features of the patients were reported and analysed. Statistical analysis was performed by SAS software version 8.0 (SAS Inc, Cary, NC, USA). Data are presented as mean (SD) unless otherwise specified. The association between baseline parameters and response to a combination of ribavirin and hydrocortisone treatment was analysed by simple logistic regression. Parameters measured during treatment were analysed using either $t$ test or Mann-Whitney test, depending on their distribution. A stepwise multiple logistic regression analysis was then performed to identify independent predictors for failure to respond to ribavirin and low dose steroid, which would lead to the subsequent use of pulse methylprednisolone in accordance with our treatment protocol. All clinical parameters with a $\mathrm{p}$ value of $<0.20$ by univariate analysis were entered into the model. A p value of $<0.05$ was considered statistically significant.

\section{RESULTS}

In the 2 week period from 11 March to 25 March 2003 a total of 156 patients were admitted to the Prince of Wales Hospital with SARS, of whom 138 were identified as either secondary or tertiary cases stemming from our index patient. The demography of this cohort has been described in detail in a previous report. ${ }^{1}$ Briefly, there were 66 men and 72 women with a mean (SD) age of 39.3 (16.8) years. The duration between onset of symptoms and admission ranged from 0 to 11 days (median 3 days). Of these 138 cases, 124 subsequently showed seroconversion to SARS CoV while two had negative SARS CoV serology. For the remaining 12, convalescent serum was not obtained but eight had SARS CoV isolated from the nasopharyngeal aspirate $(n=3)$ or necroscopic lung and/or intestinal tissues $(n=5)$. Thus, only in four patients was SARS CoV infection status not established.

None of the 138 cases responded to antibiotics. Fever persisted in all and lung consolidation progressed in the majority of patients. Ninety four patients received oral ribavirin and prednisolone, of which 14 were sustained 
responders and nine were partial responders. These 23 patients were discharged uneventfully. Two patients died in the early phase of the disease before additional treatment could be given. Forty four patients received intravenous ribavirin and hydrocortisone, two of whom had a sustained response and four died. The remaining 107 patients showed no appreciable response to this combination treatment: 11 had persistent fever, 51 had radiological deterioration and developed respiratory insufficiency, and 45 had both fever and respiratory deterioration (fig 2). Overall, 25 patients (18.1\%) responded to ribavirin and low dose corticosteroid treatment alone (table 1 ).

Intravenous pulse methylprednisolone was given to 107 patients who did not respond to ribavirin and low dose corticosteroid therapy. After three infusions of $0.5 \mathrm{~g}$ per dose of methylprednisolone, 45 patients $(42.1 \%)$ showed a sustained response with recovery from the disease and 52 $(48.6 \%)$ had a partial response. Thirty one of those with a partial response recovered and were discharged from hospital, one died, and 20 required further pulses of high dose methylprednisolone. There were 10 non-responders, one of whom died. Among the partial responders and nonresponders, 29 received further doses of intravenous methylprednisolone up to $3 \mathrm{~g}$ in total. A sustained response was observed in five and a partial response in 13. Eleven patients (median age 55 years, range 33-82) failed to show any response after more than three pulses of high dose methylprednisolone (fig 2). Of these, six died, one (who

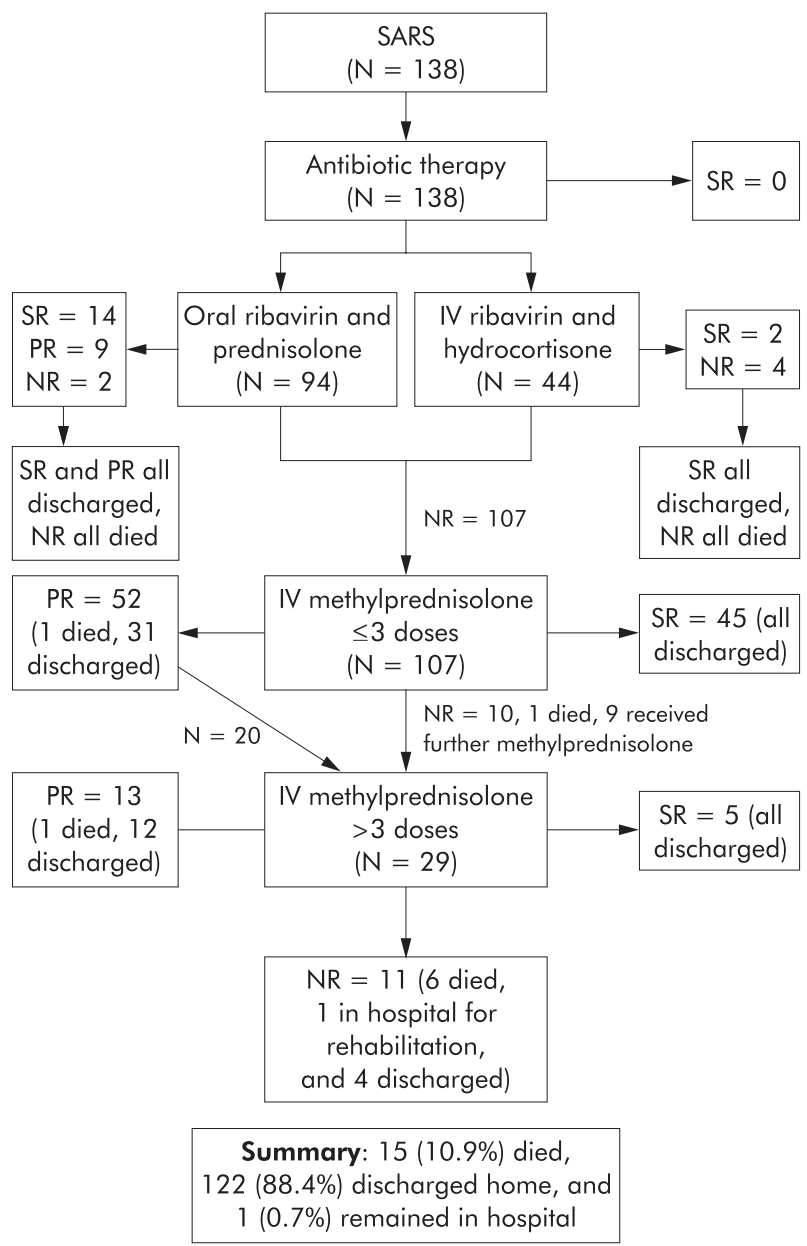

SR: sustained response, PR: Partial response, NR: No response

Figure 2 Clinical outcome of 138 patients with SARS (at 28 July 2003). has required invasive mechanical ventilation for 3 months) remains in hospital for rehabilitation, and four were discharged on a course of oral prednisolone at $0.5 \mathrm{mg} / \mathrm{kg}$ after 3 weeks of hospitalisation. The overall success rate of high dose methylprednisolone treatment was $88.8 \%$.

As of 28 July 2003, there were 15 deaths (mortality rate $10.9 \%)$. The median age of these 15 patients was 69 years (range 44-82). Two (aged 68 and 69 years) had an unremarkable past history while 13 had at least one chronic medical co-morbidity (including ischaemic heart disease, rheumatic heart disease, congestive heart failure, myelodysplastic syndrome with malignant transformation, alcoholic liver cirrhosis, reactivation of viral hepatitis B infection, chronic renal impairment, and diabetes mellitus).

We have noted a typical clinical course of SARS. Fever subsides after patients receive ribavirin and low dose corticosteroid in the first week. While some patients remain in remission, almost $80 \%$ of patients have recurrence of fever with radiological progression by the second week. This is accompanied by the development of respiratory symptoms and hypoxaemia. Intravenous high dose methylprednisolone usually controls the fever and results in resolution of lung shadows and improvement in oxygenation. Biochemical parameters and blood counts take $2-3$ weeks to return to normal (fig 3).

Hyperglycaemia (plasma spot glucose $\geqslant 11.0 \mathrm{mmol} / \mathrm{l}$ ) was detected in 23 of the 107 patients and hypokalaemia (plasma potassium level $\leqslant 3.0 \mathrm{mmol} / \mathrm{l}$ ) in 16 . These metabolic derangements were reversed when intravenous high dose methylprednisolone was discontinued. Two patients developed transient confusion, delusion, and anxiety. CT scanning and MR imaging of the brain revealed no abnormalities, and the EEG and cerebrospinal fluid analysis were also normal. The symptoms gradually subsided after discontinuation of steroid.

The 25 patients who responded to ribavirin and low dose steroid were compared with the 113 patients who did not respond and needed further treatment with pulse methylprednisolone (table 2). Initial platelet count, initial LDH, systolic blood pressure, and initial CRP level reached statistical significance by univariate analysis. Multivariate analysis showed that a higher initial level of CRP (odds ratio per $10 \mathrm{mg} / \mathrm{dl}, 2.18 ; 95 \%$ confidence interval 1.12 to 4.25 ; $p=0.02$ ) was the only independent risk factor for the use of pulse methylprednisolone. Among the parameters recorded during treatment, those who failed to improve after ribavirin and low dose steroid had a significantly lower nadir lymphocyte count and higher peak LDH and CRP levels.

Thirty seven patients $(26.8 \%)$ were admitted to the ICU. Of these, $21(15.2 \%)$ required endotracheal intubation and mechanical ventilation. Barotrauma was noted in eight patients (21.6\% of ICU admissions). Pneumomediastinum with subcutaneous surgical emphysema was seen in three cases and pneumothorax in five. In one case the pneumothorax occurred within 24 hours of the insertion of a central venous catheter in the internal jugular vein. Nosocomial infection was diagnosed in 17 of the 37 patients admitted to the ICU. ${ }^{90}$ In six the diagnosis of nosocomial infection was made before high dose methylprednisolone administration. Infections included pneumonia in 10 patients (MRSA in six, Stenotrophomonas maltophilia in two, Candida albicans in one, and polymicrobial in one), urinary tract infections in two patients (Escherichia coli in one and Enterococcus spp in one), and bacteraemia with no clearly identified site in five patients (MRSA in three, Enterococcus spp in one, and Candida albicans in one). Clostridium difficile toxin was identified in one patient with diarrhoea. Sepsis induced organ failure was considered to have contributed to death in five cases. 
Table 1 Clinical response to treatment

\begin{tabular}{|c|c|c|c|}
\hline & $\begin{array}{l}\text { Broad spectrum } \\
\text { antimicrobial }^{*}(n=138)\end{array}$ & $\begin{array}{l}\text { Ribavirin + corticosteroidt } \\
(n=138)\end{array}$ & $\begin{array}{l}\text { IV methylprednisoloneł } \\
(\mathrm{n}=107)\end{array}$ \\
\hline Sustained response & $0(0)$ & 16 (11.6\%) & $50(46.7 \%)$ \\
\hline Partial response & $0(0)$ & $9(6.5 \%)$ & 45 (42.1\%) \\
\hline No response & $138(100 \%)$ & $113(81.9 \%)$ & 12 (11.2\%) \\
\hline \multicolumn{4}{|c|}{$\begin{array}{l}\text { *Antimicrobials included cefotaxime and clarithromycin (or levofloxacin) +oseltamivir. } \\
\text { †Ribavirin (oral or intravenous) + oral prednisolone or intravenous hydrocortisone. } \\
\text { tlntravenous methylprednisolone up to } 3 \mathrm{~g} \text { in total. } \\
\text { Clinical outcome definitions: }(1) \text { afebrile (daily peak temperature } \leqslant 37.5^{\circ} \mathrm{C} \text { ) for at least } 4 \text { consecutive days; ( } 2 \text { ) } \\
\text { resolution of chest radiograph consolidation by }>25 \% \text { (comparing film of maximal consolidation and that on the } \\
\text { 4th afebrile day); (3) oxygen independence }\left(\mathrm{SaO}_{2} \geqslant 95 \% \text { on room air) on the } 4 \text { th afebrile day. }\right. \\
\text { Sustained response }=1+2+3 \text {; partial response }=1+2 \text { or } 3 \text {; no response }=\text { failure to fulfil criteria of sustained } \\
\text { response and partial response. }\end{array}$} \\
\hline
\end{tabular}

After 2 weeks of treatment with ribavirin, 82 patients $(59 \%)$ had a fall in haemoglobin of more than $2 \mathrm{~g} / \mathrm{dl}$ from baseline while 39 (28\%) experienced a fall of more than $3 \mathrm{~g} / \mathrm{dl}$. Two weeks after initiation of ribavirin therapy haemoglobin concentrations ranged from 7.2 to $13.2 \mathrm{~g} / \mathrm{dl}$. Evidence of haemolytic anaemia was documented in 49 patients $(36 \%)$ with a rise of bilirubin $(>20 \mu \mathrm{mol} / \mathrm{l})$ and/or reticulocyte count $(>1 \%)$. No patients developed overt cardiac toxicity or renal toxicity with ribavirin.

\section{DISCUSSION}

SARS is a serious infection with a formidable morbidity and mortality. Of the 138 patients admitted to our hospital during the major outbreak, $113(81.9 \%)$ failed to improve after ribavirin and low dose corticosteroid. High dose methylprednisolone was used in 107 patients and 95 (88.8\%) responded favourably. Thirty seven patients $(26.8 \%)$ required treatment in the ICU, of whom 21 (15.2\%) required invasive mechanical ventilation. A high CRP level on admission was the only independent predictor for use of high dose methylprednisolone. As of 28 July 2003 (4 months from the outbreak onset), 122 patients $(88.4 \%)$ had been discharged and one remained in hospital for rehabilitation. The overall mortality was $10.9 \%$, most with significant co-morbidities.

Similar to the description by Peiris et al, ${ }^{11}$ the clinical course of our SARS patients appears to follow a typical pattern. Phase 1 is clinically characterised by fever, myalgia, and other systemic symptoms that generally improve after a few days. Phase 2 is characterised by recurrence of fever, oxygen desaturation, and radiological progression of pneumonia. The clinical progression during phase 2 has also been observed by others $^{11}{ }^{12}$ and appears to be related to immunopathological damage. ${ }^{11}$ Most of our patients improved during this phase with a combination of ribavirin and intravenous methylprednisolone, but $15.2 \%$ progressed into acute respiratory distress syndrome (ARDS) necessitating ventilatory support. Reports from several other series have also suggested that a substantial number of cases develop respiratory failure and

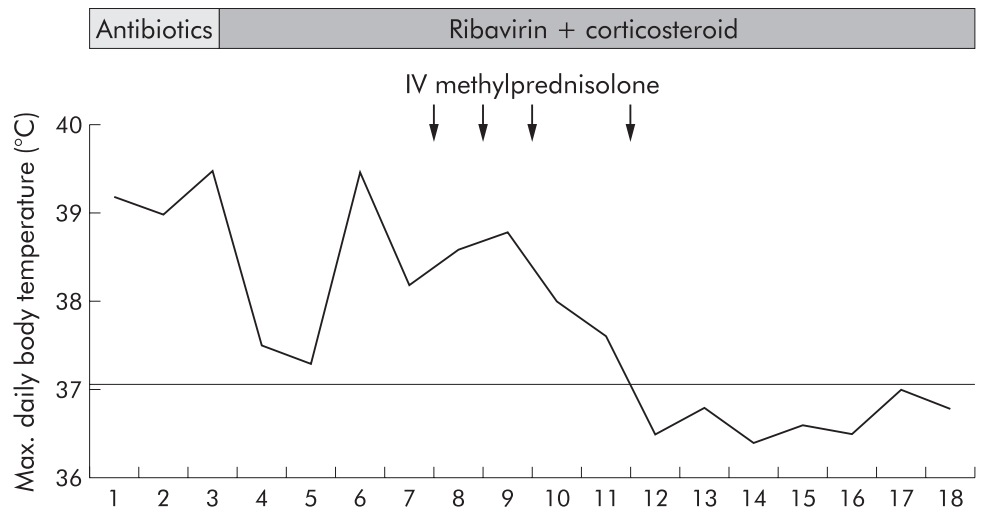

\begin{tabular}{|l|c|c|c|c|c|c|c|c|c|c|c|c|c|c|c|c|c|c|}
\hline Day & $\mathbf{1}$ & $\mathbf{2}$ & $\mathbf{3}$ & $\mathbf{4}$ & $\mathbf{5}$ & $\mathbf{6}$ & $\mathbf{7}$ & $\mathbf{8}$ & $\mathbf{9}$ & $\mathbf{1 0}$ & $\mathbf{1 1}$ & $\mathbf{1 2}$ & $\mathbf{1 3}$ & $\mathbf{1 4}$ & $\mathbf{1 5}$ & $\mathbf{1 6}$ & $\mathbf{1 7}$ & $\mathbf{1 8}$ \\
\hline $\mathrm{SaO}_{2}(\%)$ & 95 & 95 & 96 & 98 & 97 & 96 & 95 & 94 & 91 & 91 & 92 & 95 & 98 & 97 & 96 & 97 & 99 & 99 \\
\hline $\mathrm{O}_{2}$ supplement $(\mathrm{I})$ & 0 & 0 & 0 & 0 & 0 & 0 & 0 & 2 & 2 & 3 & 5 & 4 & 3 & 2 & 1 & 0 & 0 & 0 \\
\hline Neutrophil $\left(\times 10^{9}\right)$ & 7.5 & 7.7 & 9.0 & 9.8 & 9.0 & 8.8 & 16.0 & 15.1 & 19.8 & 29.8 & 23.2 & 18.3 & 17.3 & 17.2 & 16.6 & - & - & 16.3 \\
\hline Lymphocyte $\left(\times 10^{9}\right)$ & 1.7 & 1.1 & 1.1 & 1.0 & 0.9 & 0.7 & 0.5 & 0.6 & 0.7 & 0.8 & 0.5 & 0.5 & 0.7 & 0.6 & 0.5 & - & - & 0.6 \\
\hline Platelet $\left(\times 10^{3}\right)$ & 193 & 179 & 209 & 199 & 196 & 172 & 196 & 215 & 196 & 259 & 261 & 263 & 269 & 268 & 262 & - & - & 291 \\
\hline Creatinine $(\mu \mathrm{mol} / \mathrm{I})$ & 85 & 92 & 79 & 75 & 82 & 98 & 76 & 77 & 77 & 71 & 69 & 62 & 61 & 60 & 68 & - & - & 79 \\
\hline $\mathrm{ALT}(\mathrm{mmol} / \mathrm{I})$ & 18 & 18 & 16 & 21 & 19 & 16 & 23 & 31 & 40 & 40 & 60 & 73 & 68 & 62 & 86 & - & - & 54 \\
\hline $\mathrm{CPK}(\mathrm{U} / \mathrm{I})$ & 222 & 461 & 392 & 258 & 445 & 593 & 592 & 230 & 171 & 147 & 132 & 109 & 68 & 73 & 116 & - & - & 49 \\
\hline $\mathrm{LDH}(\mathrm{U} / \mathrm{I})$ & 171 & 193 & 196 & 200 & 229 & 267 & 488 & 445 & 593 & 278 & 377 & 369 & 319 & 315 & 236 & - & - & 220 \\
\hline $\mathrm{CRP}(\mathrm{mg} / \mathrm{l})$ & 4.4 & 11.9 & 7.8 & 37.4 & 60.7 & 81.6 & 83.7 & 111 & 76.7 & 42.6 & 19.9 & - & - & - & - & - & - & 0.3 \\
\hline
\end{tabular}

Figure 3 Clinical, biochemical, and haematological response of a patient to infusions of methylprednisolone. 
Table 2 Univariate analyses of clinical and laboratory parameters associated with the use of intravenous pulse methylprednisolone

\begin{tabular}{|c|c|c|c|c|}
\hline & $\begin{array}{l}\text { Not responding to } \\
\text { ribavirin+low dose } \\
\text { steroid }(n=113)^{*}\end{array}$ & $\begin{array}{l}\text { Responding to } \\
\text { ribavirin+low dose } \\
\text { steroid }(n=25)^{*}\end{array}$ & Odds ratio $(95 \% \mathrm{Cl})$ & $\mathrm{p}$ value \\
\hline \multicolumn{5}{|l|}{ Parameters at presentation } \\
\hline Age (years) & $39.9(16.4)$ & $37.5(17.8)$ & 1.01 (0.98 to 1.04$)$ & 0.5 \\
\hline Male sex $(\%)$ & $48.7 \%$ & $44.0 \%$ & $1.21(0.51$ to 2.88$)$ & 0.7 \\
\hline Duration between onset of symptom and treatment (days) & $5.4(2.7)$ & $6.1(2.8)$ & $0.91(0.77$ to 1.07$)$ & 0.2 \\
\hline Platelet $\left(\times 10^{9} / \mathrm{I}\right)$ & $146(58)$ & $175(64)$ & $0.92(0.86$ to 0.99$) \neq$ & 0.03 \\
\hline Neutrophil count $\left(\times 10^{9} / \mathrm{l}\right)$ & $4(2)$ & $3(1)$ & $1.30(0.98$ to 1.74$)$ & 0.1 \\
\hline Lymphocyte count $\left(\times 10^{9} / \mathrm{I}\right)$ & $1(1)$ & $1(1)$ & $0.82(0.45$ to 1.48$)$ & 0.5 \\
\hline Activated partial thromboplastin time (s) & $42.3(7.3)$ & $40.4(10.4)$ & $1.04(0.97$ to 1.11$)$ & 0.3 \\
\hline Sodium (mmol/l) & $135.5(3.5)$ & $136.0(2.5)$ & $0.95(0.83$ to 1.09$)$ & 0.5 \\
\hline Urea (mmol/l) & $4.8(5.4)$ & $4.4(1.1)$ & $1.02(0.91$ to 1.14$)$ & 0.7 \\
\hline Creatinine $(\mu \mathrm{mol} / \mathrm{l})$ & $101(118)$ & $86(22)$ & 1.01 (0.98 to 1.03 ) & 0.4 \\
\hline Alanine transferase (IU/I) & $28.8(30.1)$ & $62.8(158.1)$ & 1.01 (0.99 to 1.03$)$ & 0.1 \\
\hline Creatinine kinase (U/I) & $390.1(675.1)$ & $219.3(272.3)$ & $1.08(0.95$ to 1.23$) \dagger$ & 0.2 \\
\hline Lactate dehydrogenase (U/I) & 370.4 (196.5) & $262.4(114.4)$ & $1.56(1.07$ to 2.25$) \dagger$ & 0.02 \\
\hline Systolic blood pressure (mm Hg) & $117(18)$ & $109(11)$ & $1.36(1.02$ to 1.83$) \ddagger$ & 0.04 \\
\hline Diastolic blood pressure (mm Hg) & $62(11)$ & $61(11)$ & $1.07(0.72$ to 1.59$) \ddagger$ & 0.7 \\
\hline Pulse rate/min & $94(14)$ & $92(13)$ & $1.15(0.84$ to 1.57$) \ddagger$ & 0.4 \\
\hline C-reactive protein (mg/dl) & $51.9(57.2)$ & $9.6(10.3)$ & $2.18(1.12$ to 4.25$) \ddagger$ & 0.02 \\
\hline \multicolumn{5}{|l|}{ Parameters during treatment } \\
\hline Peak D-dimer (ng/ml) & $1184(1201)$ & 1247 (2119) & & 0.3 \\
\hline Lymphocyte count (nadir) $\left(\times 10^{9} /\right.$ I) & $0.3(0.2)$ & $0.6(0.4)$ & & $<0.001$ \\
\hline Creatinine kinase (peak) (U/I) & $515.7(761.4)$ & $246.4(268.8)$ & & 0.1 \\
\hline Lactate dehydrogenase (peak) (U/I) & $468.6(251.5)$ & $279.0(109.5)$ & & 0.001 \\
\hline Systolic blood pressure (nadir) (mm Hg) & $95(9)$ & $94(16)$ & & 0.6 \\
\hline Diastolic blood pressure (nadir) (mm Hg) & $48(7)$ & $49(8)$ & & 0.7 \\
\hline Pulse rate/min (peak) & $107(15)$ & $99(11)$ & & 0.01 \\
\hline C-reactive protein (peak) (mg/dl) & $82.6(83.8)$ & $13.1(15.7)$ & & $<0.001$ \\
\hline $\begin{array}{l}\text { *Values are mean (SD). } \\
\text { †Per } 100 \text { units increase. } \\
\text { †Per } 10 \text { units increase. }\end{array}$ & & & & \\
\hline
\end{tabular}

ARDS, with $17-30 \%$ of patients requiring admission to the ICU, ${ }^{11-13}$ whereas the 21 day mortality was reported to be $3.6 \%^{1}$ and $6.5 \%{ }^{13}$ respectively. Necroscopic examination revealed evidence of diffuse alveolar damage, pulmonary oedema with hyaline membrane formation, and haemophagocytosis in the lungs. ${ }^{14}$

To date there has been no consensus on the treatment of SARS. Ribavirin, a broad spectrum antiviral agent previously shown to be efficacious against both RNA and DNA viruses, has been used. ${ }^{13^{11-13}}$ Previous studies have shown that, in acute viral respiratory infections, large amounts of early response cytokines such as interferon- $\alpha$, tumour necrosis factor $\alpha$, interleukin (IL)- 1 and IL-6 are produced. These cytokines mediate antiviral activities but, at the same time, may contribute to tissue injury. ${ }^{15}$ Ribavirin can inhibit viral induced macrophage production of proinflammatory cytokines and Th2 cytokines. ${ }^{17}$ Nevertheless, it has subsequently been reported to have no significant in vitro activity against the CoV believed to be responsible for SARS. ${ }^{18}$

High dose pulse corticosteroids have been used by several groups with a favourable response. ${ }^{111}{ }^{19-22}$ Corticosteroids have been used because CT scans of the thorax have shown radiographic features of bronchiolitis obliterans organising pneumonia $(\mathrm{BOOP})^{1} 192223$ which is a steroid responsive condition suggestive of an immunological phenomenon. ${ }^{24}$ The use of high dose pulse methylprednisolone treatment aims to suppress the cytokine induced lung injury (phase 2). ${ }^{1} 112122$ A retrospective study has shown that cases receiving pulse methylprednisolone had a lower oxygen requirement, better radiographic outcome, and were less likely to require further rescue pulse steroid. ${ }^{22}$ In addition, macrophages are the prominent leucocytes in the alveoli of patients with fatal SARS, with evidence of haemophagocytosis in the lungs ${ }^{14}$ Haemophagocytosis has been attributed to cytokine dysregulation, ${ }^{25}$ and intervention with steroids might modulate this cytokine response and prevent a fatal outcome, as has been proposed for other causes of ARDS. ${ }^{14} 26$ Understandably, there are concerns about using high dose methylprednisolone in an emerging infectious disease.

In this study the treatment protocol was developed during a rapid major outbreak. The use of a relatively large dose of oral ribavirin (loading dose $2.4 \mathrm{~g}$ followed by $3.6 \mathrm{~g}$ daily) was based on the fact that the oral bioavailability of ribavirin is only $36-52 \% .{ }^{27}{ }^{28}$ High dose methylprednisolone was used only when ribavirin and low dose corticosteroid failed to stop the inflammatory process, which was evident by continuing radiological progression and hypoxaemia. Twenty five patients $(18 \%)$ responded favourably to ribavirin and low dose corticosteroid treatment. Of these, 23 received oral ribavirin and two received intravenous ribavirin; the numbers were too small to allow a meaningful comparison between them. With the dosage of ribavirin used we observed a modest degree of anaemia in most patients (in 59\% the haemoglobin fell by $2 \mathrm{~g} / \mathrm{dl}$ ), probably the result of haemolysis. A much higher dose of ribavirin, based on dosage for treatment of haemorrhagic fever viruses, ${ }^{29}$ has been reported to be associated with more significant toxicity including haemolysis (in 76\%) and a decrease in haemoglobin of $2 \mathrm{~g} / \mathrm{dl}$ (in $49 \%$ ), raised transaminases (in $40 \%$ ), and bradycardia (in $14 \%$ of SARS patients)..$^{13}$

Despite the combination of ribavirin and low dose corticosteroid, most of the patients continued to deteriorate during the second week of the illness. High dose methylprednisolone was then used in 107 patients, $95(88.8 \%)$ of whom recovered from the progressive lung disease. Following high dose methylprednisolone treatment, rapid resolution of lung opacity was usually followed by an improvement in hypoxaemia. Most patients responded after receiving three doses of methylprednisolone (up to $1.5 \mathrm{~g}$ in total). Less than $30 \%$ of cases required additional doses. The timing of 
administration of high dose methylprednisolone is important. It was administered only during phase 2 when radiological progression of consolidation and increasing hypoxaemia were documented. ${ }^{11121}$ In most cases high dose methylprednisolone was started towards the end of the first week with the first pulse administered on day 8 (median) from fever onset. We have avoided using high dose methylprednisolone in the early phase of SARS ${ }^{11}$ as viral clearance by host immunity might be hampered. It must be emphasised that high dose methylprednisolone should not be used only to control fever. In some patients the lung opacities continued to deteriorate even after defervescence. In these patients the benefit of high dose methylprednisolone in reversing radiological progression was also seen. While we recognise that the benefit of high dose methylprednisolone ${ }^{111}{ }^{19-22}$ cannot be confirmed without a control group, the use of high dose corticosteroid in the treatment of SARS warrants further investigation.

Our analysis indicated that patients with thrombocytopenia, high LDH, and high CRP levels at presentation were more likely to have uncontrolled inflammation requiring high dose corticosteroid therapy. Of these, a high CRP level at presentation was the only independent factor of nonresponse to ribavirin and low dose corticosteroid. During treatment with ribavirin and low dose corticosteroid the nonresponders also had more severe lymphopenia, a higher peak $\mathrm{LDH}$, and a higher peak CRP level. While a high peak LDH is a poor prognostic marker ${ }^{1}$ and is most likely the result of immune mediated lung injury in severe cases of SARS, ${ }^{11}$ serial measurements of CRP may be useful in monitoring response to treatment and detecting complications in patients with secondary infections. ${ }^{30} 31$

In those patients admitted to the ICU the predominant feature was isolated respiratory failure. All other recorded organ failure was either pre-existing or could be attributed to nosocomial infections. Despite low volume, low pressure mechanical ventilation, the incidence of barotrauma $(21.6 \%$ of our ICU admissions) was surprisingly high. Chest radiographs and CT scans did not indicate excessive hyperinflation or bullous lung disease and, at present, we can offer no explanation for this observation other than poor lung compliance. ${ }^{23}$ Prone ventilation as salvage therapy appears to have benefited some patients, but its use is controversial in view of the lack of evidence supporting any benefit on mortality. ${ }^{32}$ The unusually high rate of nosocomial infection, particularly pneumonia, may have been the consequence of corticosteroid treatment. More serious complications such as disseminated fungal disease have been reported elsewhere. ${ }^{33}$

This report provides an account of a stepwise approach to the treatment of SARS. The use of ribavirin has led to a significant degree of haemolytic anaemia and the lack of in vitro antiviral activity of ribavirin against SARS $\mathrm{CoV}^{18}$ has rendered its role doubtful in the treatment of SARS. The use of high dose methylprednisolone during clinical progression, on the rationale of preventing immunopathological lung injury, ${ }^{11} 14212226$ appeared to be effective in our cohort but the limitation of interpreting uncontrolled data should be noted. Randomised controlled studies will be required to evaluate the efficacy and best timing for high dose methylprednisolone treatment. It is hoped that the availability of the genome sequence of the SARS $\operatorname{CoV}^{34-36}$ will facilitate efforts to develop new and rapid diagnostic tests, antiviral agents, and vaccines in the long run.

\section{ACKNOWLEDGEMENTS}

The authors would like to thank all the healthcare workers who have participated in the battle against SARS. In addition, they would like to thank Ms MY Yung and Mr Michael Chung for helping with data entry and analysis.

\section{Authors' affiliations}

J J Y Sung, A Wu, N Lee, C S Cockram, V W Wong, D S C Hui, Department of Medicine and Therapeutics, The Chinese University of Hong Kong, Hong Kong

G M Joynt, Department of Anesthesia and Intensive Care, The Chinese University of Hong Kong, Hong Kong

K Y Yuen, Department of Microbiology, The University of Hong Kong, Hong Kong

P K S Chan, Department of Microbiology, The Chinese University of Hong Kong, Hong Kong

A T Ahuja, Department of Diagnostic Radiology and Organ Imaging, The Chinese University of Hong Kong, Hong Kong

L M Yu, Center for Clinical Trial and Epidemiological Research, The Chinese University of Hong Kong, Hong Kong

\section{REFERENCES}

1 Lee N, Hui DS, Wu A, et al. A major outbreak of severe acute respiratory syndrome in Hong Kong. N Engl J Med 2003;348:1986-94.

2 Update: outbreak of severe acute respiratory syndrome-worldwide 2003. MMWR Morb Mort Wkly Rep 2003;52:241-8.

3 Kuiken T, Fouchier RA, Schutten M, et al. Newly discovered coronavirus as the primary cause of severe acute respiratory syndrome. Lancet 2003;362:263-70.

4 Drosten C, Gunther S, Preiser W, et al. Identification of a novel Coronavirus in patients with severe acute respiratory syndrome. N Engl J Med 2003:348:1967-76.

5 Ksiazek TG, Erdman D, Goldsmith CS, et al. A Novel Coronavirus associated with severe acute respiratory syndrome. N Engl J Med 2003;348:1953-66.

6 Centers for Disease Control and Prevention. Severe acute respiratory syndrome (SARS) updated interim U.S. case definition. http://www.cdc.gov/ ncidod/sars/casedefinition.htm (accessed 20 April 2003).

7 Guidelines for the management of adults with community-acquired pneumonia: diagnosis, assessment of severity, antimicrobial therapy, and prevention. Am J Respir Crit Care Med 2001;163:1730-54.

8 The Acute Respiratory Distress Syndrome Network. Ventilation with lower tidal volumes as compared with traditional tidal volumes for acute lung injury and the acute respiratory distress syndrome. N Engl J Med 2000;342:1301-8.

9 American Thoracic Society. Hospital-acquired pneumonia in adults: diagnosis, assessment of severity, initial antimicrobial therapy, and preventive strategies. A consensus statement. Am J Respir Crit Care Med 1995; 153:1711-25.

10 Garner JS, Jarvis WR, Emori TG, et al. CDC definitions for nosocomial infections. Am J Infect Control 1988;16:128-40.

11 Peiris JS, Chu CM, Cheng VC, et al. Clinical progression and viral load in a community outbreak of coronavirus-associated SARS pneumonia: a prospective study. Lancet 2003;361:1767-72.

12 Hsu LY, Lee CC, Green JA, et al. Severe acute respiratory syndrome in Singapore: clinical features of index patient and initial contacts. Emerg Infect Dis 2003;9:713-7.

13 Booth CM, Matukas LM, Tomlinson GA, et al. Clinical features and short-term outcomes of 144 patients with SARS in the greater Toronto area. JAMA 2003;289:2801-9.

14 Nicholls JM, Poon LL, Lee KC, et al. Lung pathology of fatal severe acute respiratory syndrome. Lancet 2003;361:1773-8.

15 Cheung CY, Poon LL, Lau AS, et al. Induction of proinflammatory cytokines in human macrophage by influenza $A(\mathrm{H} 5 \mathrm{~N} 1)$ virus: a mechanism for the unusual severity of human disease? Lancet 2002;360:1831-7.

16 Van Reeth K, Van Gucht S, Pensaret M. In vivo studies on cytokines involvement during acute viral respiratory infection of swine: troublesome but rewarding. Vet Immun Immunopathol 2002;87:161-8.

17 Ning Q, Brown D, Parodo J, et al. Ribavirin inhibits viral-induced macrophage production of TNF, IL-1, the procoagulant $\mathrm{fg} 12$ prothrombinase and preserves Th1 cytokine production but inhibits Th2 cytokine response. J Immunol 1998; 160:3487-93.

18 Cyranoski D. Critics slam treatment for SARS as ineffective and perhaps dangerous. Nature 2003;423:4

19 Tsang KW, Ho PL, Ooi GC, et al. A cluster of cases of severe acute respiratory syndrome in Hong Kong. N Engl J Med 2003;348:1977-85.

20 So LK, Lau AC, Yam LY, et al. Development of a standard treatment protocol for severe acute respiratory syndrome. Lancet 2003;361:1615-7.

21 Nhong NS, Zeng GQ. Our strategies for fighting severe acute respiratory syndrome. Am J Respir Crit Care Med 2003;168:7-9.

22 Ho JC, Ooi GC, Mok TY, et al. High dose pulse versus non-pulse corticosteroid regimens in severe acute respiratory syndrome. Am J Respir Crit Care Med 2003, Epub ahead of print URL: http://ajrccm.atsjournals.org/cgi/reprint/ 200306-7660Cv1.pdf

23 Wong KT, Antonio GE, Hui DS, et al. Thin section CT of severe acute respiratory syndrome: evaluation of 73 patients exposed to or with the disease. Radiology 2003;228:395-400.

24 Epler GR. Bronchiolitis obliterans organizing pneumonia. Arch Intern Med $2001 ; 161: 158-64$

25 Fisman DN. Hemophagocytic syndrome and infection. Emerg Infect Dis 2000;6:60-8.

26 Lai KN, Leung JC, Metz CN, et al. Role for macrophage migration inhibitory factor in acute respiratory distress syndrome. J Pathol 2003; 199:496-508. 
27 Laskin OL, Longstreth JA, Hart CC, et al. Ribavirin disposition in high-risk patients for acquired immunodeficiency syndrome. Clin Pharm Ther 1987;41:543-55

28 Roberts RB, Laskin OL, Laurence J, et al. Ribavirin pharmcodynamics in highrisk patiens for acquired immunodeficiency syndrome. Clin Pharm Ther 1987;42:365-73.

29 Borio L, Inglesby T, Peters CJ, et al. Hemorrhagic fever viruses as biological weapons: medical and public health management. JAMA 2002;287:2391-405.

30 Hansson LO, Lindquist L. C-reactive protein: its role in the diagnosis and follow-up of infectious diseases. Curr Opin Infect Dis 1997;10:196-201.

31 Gabay C, Kushner I. Mechanisms of disease: acute-phase proteins and other systemic responses to inflammation. N Engl J Med 1999;340:448-54.
32 Gattinoni L, Tognoni G, Pesenti A, et al. Effect of prone positioning on the survival of patients with acute respiratory failure. N Engl J Med 2001;345:568-73.

33 Wang $H$, Ding Y, Li X, et al. Fatal aspergillosis in a patient with SARS who was treated with corticosteroids. N Engl J Med 2003;349:507-8.

34 Rota PA, Oberste MS, Monroe SS, et al. Characterization of a novel coronavirus associated with severe acute respiratory syndrome. Science 2003;300:1394-9

35 Marra MA, Jones SJ, Astell CR, et al. The genome sequence of the SARSassociated coronavirus. Science 2003;300:1399-404

36 Ruan YJ, Wei CL, Ee LA, et al. Comparative full-length genome sequence analysis of 14 SARS coronavirus isolates and common mutations associated with putative origins of infection. Lancet 2003;361:1779-85.

\section{LUNG ALERT}

Chronic occupational exposure to nitrogen dioxide is associated with decline in lung function

$\Delta$ Bakke B, Ulvestad B, Stewart P, Eduard W. Cumulative exposure to dust and gases as determinants of lung function decline in tunnel construction workers. Occup Environ Med 2004;61:262-9

ix hundred and fifty one Norwegian male construction workers were followed for a

$\mathrm{S}$ mean of 6 years with spirometric measurement and assessment of their occupational exposure to total dust, respirable dust, $\alpha$-quartz, volatile organic compounds, oil vapour, oil mist, formaldehyde, nitrogen dioxide, and carbon monoxide. Compared with a low exposure reference group of outdoor (non-tunnel) workers, tunnel workers showed a decrease in lung function between the first and last spirometric assessments. The excess annual decline in forced expiratory volume in 1 second $\left(\mathrm{FEV}_{1}\right)$ in non-smoking tunnel construction workers was $26 \mathrm{ml}$ for drill and blast workers, $31 \mathrm{ml}$ in tunnel concrete workers, and $21 \mathrm{ml}$ in shotcreters. The decrease in $\mathrm{FEV}_{1}$ was found to be significantly associated with all exposures. Multiple linear regression modelling for decline in $\mathrm{FEV}_{1}$ showed that cumulative exposure to nitrogen dioxide had the strongest association of all the agents.

This study demonstrates a decline in lung function in tunnel construction workers exposed to nitrogen dioxide and other agents over a 6 year period. It highlights a need for decreasing occupational exposure to nitrogen dioxide, and also to respirable dust and $\alpha$ quartz, which could be achieved by reducing diesel exhaust emissions and deployment of respiratory devices to protect workers.

R Johns MRC Clinical Research Fellow, University College, London, UK r.johns@ucl.ac.uk 